\title{
Modulation of spatial Stroop by object-based attention but not by space-based attention
}

\author{
Chunming Luo \\ State Key Laboratory of Brain and Cognitive Science, Institute of Psychology, and Graduate University of the Chinese Academy of Sciences, \\ Chinese Academy of Sciences, Beijing, China \\ Juan Lupiáñez and María Jesús Funes \\ University of Granada, Spain \\ Xiaolan Fu \\ State Key Laboratory of Brain and Cognitive Science, Institute of Psychology, Chinese Academy of Sciences, Beijing, China
}

\begin{abstract}
Earlier studies have shown that the spatial Stroop effect systematically decreases when a peripheral precue is presented at the same location as the target, compared to an uncued location condition. In this study, two experiments were conducted to explore whether the cueing modulation of spatial Stroop is object based and/or space based. In Experiment 1, we found evidence favouring the view that the cueing modulation of the spatial Stroop effect is entirely object based, as no differences were found in conflict reduction for the same-location and same-object conditions. In Experiment 2, the cue was predictive, and a similar object-based modulation of spatial Stroop was still observed. However, the direction of such modulation was affected by the rectangles' orientation. Overall, the pattern of results obtained favours the object-integration (Lupiáñez \& Milliken, 1999; Lupiáñez, Milliken, Solano, Weaver, \& Tipper, 2001) and referential-coding accounts (Danziger, Kingstone, \& Ward, 2001) and seems to provide evidence against the attention-shift account (Rubichi, Nicoletti, Iani, \& Umilta, 1997; Stoffer, 1991).
\end{abstract}

Keywords: Object-based attention; Space-based attention; Spatial Stroop effect; Spatial code.

One of the most widely studied perception-action relations concerns how the locations of objects in space are coded and how actions are organized on the basis of these representations (Rubichi, $\mathrm{Vu}$, Nicoletti, \& Proctor, 2006). One of the main tools to study this spatial coding is to

Correspondence should be addressed to Xiaolan Fu, State Key Laboratory of Brain and Cognitive Science, Institute of Psychology, Chinese Academy of Sciences, 4A Datun Road, Chaoyang District, Beijing 100101, China. E-mail: fuxl@psych.ac.cn

This research was supported in part by grants from the 973 Program of the Chinese Ministry of Science and Technology (Grant No. 2006CB303101) and the National Natural Science Foundation of China (Grants Nos. 60433030, 30500157, and 30600182). Financial support was also provided by the Spanish Ministry of Education and Science with research projects PSI2008-03595PSIC, PSI2008-04223PSIC, and CSD2008-00048. We wish to thank Robert W. Proctor, Bernhard Hommel, Qiufang Fu, Nachshon Meiran, Avishai Henik, and two anonymous reviewers for their helpful comments on the manuscript. The research is submitted in partial fulfilment of the requirements of a $\mathrm{PhD}$ for Chunming Luo.

\section{6}

(C) 2009 The Experimental Psychology Society

http://www.psypress.com/qjep 
examine spatial congruency effects. This approach suggests that the spatial location of an object is automatically coded, as it usually has an influence on performance even when the location is completely irrelevant for the task. Two well-known spatial congruency paradigms are the Simon effect and the spatial Stroop effect (Lu \& Proctor, 1994; O'Leary \& Barber, 1993; Walley, McLeod, \& Weiden, 1994; see Lu \& Proctor, 1995, for a review).

As an example of the spatial Stroop task, a left-pointing or right-pointing arrow target is presented randomly to the left or right side of a fixation point. Although participants are required to discriminate the direction of the arrow while ignoring its location, they typically give faster and more accurate responses to congruent stimuli (i.e., a right-pointing arrow on the right) than to incongruent ones (i.e., a left-pointing arrow on the right; Funes, Lupiáñez, \& Milliken, 2007; Lupiáñez \& Funes, 2005; Taylor \& Ivanoff, 2005). The spatial Stroop effect seems to reflect a conflict between two streams of stimulus information when the irrelevant stimulus dimension (location information) is incongruent with its relevant dimension (the direction of the arrow; Lu \& Proctor, 1995).

Since responses are systematically affected by target location, spatial congruency effects have been used to study the influence of irrelevant spatial information (location) and have been interpreted as a direct index of the formation of spatial codes (e.g., Danziger, Kingstone, \& Ward, 2001; Lupiáñez \& Funes, 2005).

Besides, there is general interest to determine whether attention plays a special role in spatial stimulus coding (Hommel, 1993b; Lu \& Proctor, 1995). Consequently, several studies have been carried out to determine whether spatial cueing manipulations can modulate the size of spatial congruency effects such as the Simon effect (e.g., Hommel, 1993a; Proctor, Lu, \& van Zandt, 1992; Zimba \& Brito, 1995) or the spatial Stroop effect (e.g., Danziger, Kinstone, \& Ward, 2001; Funes \& Lupiáñez, 2003; Funes et al., 2007; Funes, Lupiáñez, \& Milliken, 2008; Lupiáñez \& Funes, 2005). The general idea is to explore whether spatial congruency effects are different at attended or cued locations versus unattended or uncued locations. Several predictions have been made by various accounts.

According to the attention-shift account (Rubichi, Nicoletti, Iani, \& Umilta, 1997; Stoffer, 1991), attention shifts generate spatial codes relative to the prior position of attention. Therefore, if attention has been moved towards the cued location prior to target appearance, no attentional shift toward the target location will be necessary when the target is presented. Therefore, no spatial code will be created for the target, and consequently a null Simon or spatial Stroop effect should be observed on cued trials. Yet, contrary to this prediction, several studies have observed that the Simon effect was not smaller at attended locations than at unattended ones (e.g., Hommel, 1993b; Ivanoff, Klein, \& Lupiáñez, 2002; Proctor et al., 1992; Verfaellie, Bowers, \& Heilman, 1988; Weeks, Chua, \& Hamblin, 1996; Zimba \& Brito, 1995).

According to the referential-coding account (Hommel, 1993b), the location of a stimulus tends to be coded in terms of its position relative to an environmental object of reference, such as the central fixation point in a spatial cueing paradigm (e.g., environmental coordinates). In this view, the occurrence of Simon-like effects is not bound to any attentional movement toward the stimulus location. Consequently, the orienting of attention triggered by spatial cues should not modulate spatial congruency effects such as the Simon effect. However, a new version of the referential-coding account has been recently proposed by Danziger et al. (2001). According to them, the target spatial location may be right/left coded relative to multiple reference objects. Within the context of the spatial cueing paradigm, spatial cues may constitute mere objects of reference for the creation of target spatial coordinates (Danziger et al., 2001). More specifically, the target spatial location may be right/left coded relative to two simultaneous objects of reference-the central fixation point object and the lateralized cue object. In opposite-cued trials, the target location is coded relative to both the left/right cued 
location and the central location. In cued trials, however, the target location is right/left coded only relative to the central point, because it would be coded as "same" relative to the cue. This explanation is expected to predict a reduction of Simon-like effects in cued trials compared to opposite-cued trials.

Congruent with this prediction, a number of recent studies have observed a systematic reduction of spatial Stroop in cued trials compared to uncued ones (Danziger et al., 2001; Funes \& Lupiáñez, 2003; Funes et al., 2007; Lupiáñez \& Funes, 2005).

Although the multiple referential-coding account proposed by Danziger et al. (2001) fits well with some findings within the context of the spatial Stroop paradigm, it cannot explain other related findings. For example, in Funes and Lupiáñez's (2003) study, peripheral noninformative cues were presented in two thirds of the trials. No cues were presented in the remaining third of trials. In half of the cued trials, the cue directed attention to the target location; in the other half, it directed attention to the location opposite the target. Spatial cues modulated the spatial congruency effect, as the size of spatial congruency was significantly smaller in cued-location trials $(27 \mathrm{~ms})$ than in no-cue trials $(44 \mathrm{~ms})$ and greater in opposite-cued-location ones $(58 \mathrm{~ms}$ ). This finding cannot be explained by Danziger et al.'s (2001) referential-coding account, which predicts a similar congruency effect in cued and no-cue trials, as the targets would be spatially coded only relative to the central fixation point.

Moreover, in Lupiáñez and Funes (2005, Experiment 2), participants were instructed to discriminate the direction of an arrow that could appear in any of four locations: left, right, top, or bottom. The arrow could point either up or down, and the participants were to hit either the left or right key depending on the direction of the arrow. Thus, when the arrow appeared on the vertical axis (top/bottom locations) a pure measure of spatial location-direction congruency or spatial Stroop (stimulus-stimulus, S-S) was obtained, given that the responding hand (left or right) was orthogonal to the location and direction of the arrow (top/bottom, up/down). When the arrow appeared on the horizontal axis (left/right), however, a pure measure of stimulus-response $(\mathrm{S}-\mathrm{R})$ spatial congruency or Simon effect was obtained, given that the direction of the arrow (up or down) was orthogonal to the responding hand and location of the arrow (left or right). Targets were preceded by an nonpredictive spatial cue to guide attention to one of the four locations. It is important to note that spatial cues significantly modulated the spatial Stroop effect (stimulusstimulus correspondence; the effect was lower in cued location trials), whereas they did not modulate the Simon effect (stimulus-response correspondence). Danziger et al.'s (2001) referential-coding account cannot explain why peripheral cueing did not modulate the Simon effect in this study and others described above (Hommel, 1993b; Ivanoff et al., 2002; Proctor et al., 1992; Verfaellie et al., 1988; Weeks et al., 1996; Zimba \& Brito, 1995). Indeed, the target location should be coded relative to an equivalent set of objects of reference in uncued trials (relative to the left/right cue and the central fixation point) and cued trials (relative only to the fixation point) in both spatial Stroop and Simon paradigms. This asymmetry in the modulation of the Simon and spatial Stroop effects by peripheral cueing is also at odds with the attentional shift account, which predicts a reduction of any kind of spatial congruency effects in cued trials.

Finally, Funes et al. (2007; Experiment 2) have recently found that the decrease of spatial Stroop in cued trials is independent of the predictive value of the peripheral cue. In fact, the same decrease was obtained following noninformative peripheral cues and peripheral cues that were highly predictive about the target location, in spite of predictive cues producing greater orienting effects. This finding suggests that an explanation in terms of attention shift might not be sufficient to account for such an effect. Considering the whole set of data about the spatial cueing modulation of the spatial Stroop effect (independence of cue predictability, specificity to $\mathrm{S}-\mathrm{S}$ congruency), the authors proposed that such pattern of modulation could be better explained in terms 
of Lupiáñez et al.'s object-file integration account (Lupiáñez \& Milliken, 1999; Lupiáñez, Milliken, Solano, Weaver, \& Tipper, 2001).

According to this account, an abrupt onset (peripheral cue) can be regarded as a perceptual object or event (Jonides \& Yantis, 1988; Yantis \& Jonides, 1996) that shares spatial location with the target and is contiguous in time with it. Assuming that spatial and temporal contiguity play an important role in event- or objectintegration processes (Kahneman, Treisman, \& Gibbs, 1992), the facilitation effect often observed at short cue-target stimulus onset asynchronies (SOAs) could be attributed - at least partly - to rapid integration of the spatial codes for the cue and the target when they occur close together in both time and space (see Funes \& Lupiáñez, 2003; Funes, Lupiáñez, \& Milliken, 2005; Funes et al., 2007, 2008; Lupiáñez \& Funes, 2005; Lupiáñez \& Milliken, 1999; Lupiáñez et al., 2001, for discussions of event-integration processes in exogenous cueing contexts). These authors assume that the integration of cue and target spatial codes within the same event or object file prevents any extra spatial codes from being created when the target appears. This integration process thus helps separate the processing of the two conflicting dimensions of the target stimulus - the spatial location and its directionin time; the distracting location dimension of the arrow target is linked to an event that occurred at an earlier point in time (the cue). The separation in time of these two perceptual codes may underlie the decrease in the spatial congruency effect observed in validly cued trials, as the irrelevant location dimension should have largely declined by the time the relevant direction dimension is coded (see Hommel, 1993a, for a discussion of this temporal overlap hypothesis as it applies to Simon interference). It is worth noting that that cue-target event integration is not believed to occur when the cue and target appear at different locations, as would be the case for uncued trials following peripheral noninformative cues, and for no-cue trials (Funes \& Lupiáñez, 2003).

According to the object-file integration account, the object occupying the cued location (the box marker) is also cued. Thus, the cueing modulation on the spatial Stroop effect may be mainly due to the cueing of the object (instead of the location) in which the target appears. However, in the context of the standard cueing paradigm used in the spatial Stroop studies described above, the cued location always corresponded to the object location. This makes it impossible to elucidate whether the decrease in spatial congruency is due to the cueing of the target location or of the object in which it appears.

In fact, there is growing evidence showing that attention may have two underlying componentsspace-based and object-based ones - at least when peripheral cues are used (e.g., Egly, Driver, \& Rafal, 1994; Goldsmith \& Yeari, 2003; Weaver, Lupiáñez, \& Watson, 1998). In the original double-rectangle cueing procedure, two parallel rectangles-one at either side of the fixation point-were vertically or horizontally presented; participants were required to detect a small target, which would appear at one end of one of the rectangles. Shortly before the target onset, the end of one of the rectangles was briefly flashed as a cue. In $75 \%$ of the trials (valid-cue trials), the target was presented at the cued location. In the remaining trials, the target appeared at one of two locations, equally distant from the cued location: (a) at the opposite end of the same rectangle (same-object trials) or (b) at the nearer end of the other rectangle (differentobject trials). Egly et al. (1994) found that target detection was faster in validly cued trials than in invalidly cued ones, suggesting that location or distance from the cue affected performancespace-based effect. In addition, when invalid-cue trials were examined separately, target detection was faster for same-object targets than for different-object targets, notwithstanding their equivalent distance from the cued location. This suggests that the rectangle also influenced the allocation of attention - an object-based effect.

The object-file integration framework developed by Lupiáñez and Milliken to account for spatial cueing effects (Lupiáñez \& Milliken, 1999; Lupiáñez et al., 2001) and their modulation of the spatial Stroop effect (Funes et al., 2008; 
Lupiáñez \& Funes, 2005) provides considerable inspiration for further studies. To our knowledge, however, not much research has been conducted to directly test the relations between object-based attention and spatial coding, which is the main goal of this paper.

\section{EXPERIMENT 1}

In this experiment, participants responded to a left/right pointing arrow whose direction was to be discriminated (see Figure 1). The arrow target could appear at one end of one of two rectangles shown vertically or horizontally (see Figure 1). Unlike the case in Egly et al. (1994), the cue was not informative - that is, its appearance at one of the four possible locations (the two ends of the two rectangles) was equiprobable. Thus, we combined the double-rectangle cueing paradigm developed by Egly et al. (1994) with the spatial Stroop task used by Lupiáñez and Funes (2005) to distinguish the role of pure object-based and space-based attention in the generation and modulation of spatial codes. The use of this paradigm will allow us to directly test the three main hypotheses described in the introduction to account for the reduction of spatial congruency effects by cueing. If the reduction of spatial Stroop by cueing observed in previous studies arises from event- or object-integration processes, we expect to find such reduction to be merely object based. Consequently, the reduction of spatial Stroop will not differ between samelocation and same-object conditions. In contrast, if such an effect is attributed to attention shifts, we expect the two components of attention to jointly modulate the spatial Stroop effect. Consequently, the reduction of spatial Stroop either will take place exclusively in the samelocation condition or will be stronger in this condition than in the same-object condition.

Finally, according to the referential-coding account (Danziger et al., 2001), we expect the modulation of spatial Stroop to be object based but dependent on the arrangement of the rectangles. For vertically arranged rectangles, we expect a similar reduction of spatial Stroop in the same-object and same-location conditions. In the case of different-object trials, the target location should be coded relative to both the left/right cued location and the central location; however, in same-location and same-object trials, the target location should be left/right coded only relative to the central fixation point, because it would be coded as "same" relative to the cue in both cases. However, for horizontally arranged rectangles, we expect an increase in spatial Stroop for same-object compared to same-location and different-object conditions. In this case, in contrast with that of vertically arranged rectangles, the target location is expected to be left/right coded only relative to the central fixation point in the same-location and different-object

\section{STIMULUS FIELDS}

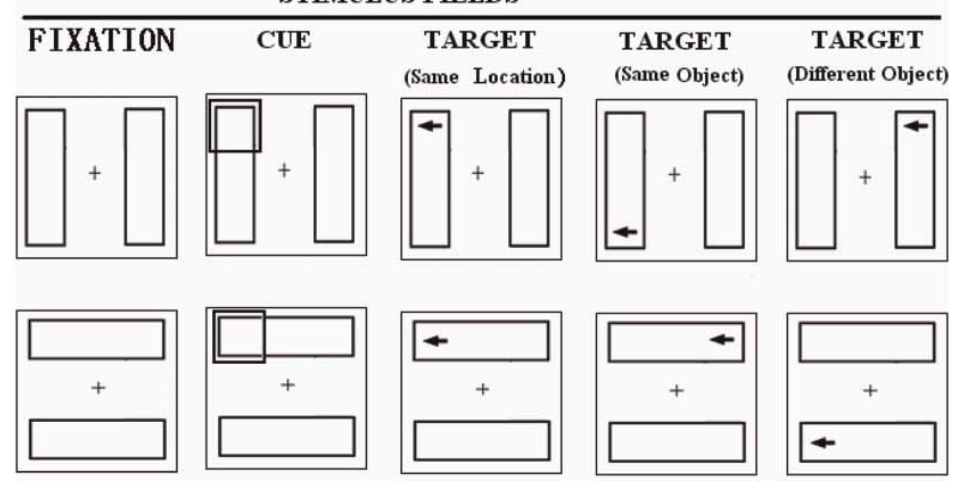

Figure 1. Examples of the typical sequences of events within trials from the major conditions of Experiment 1. 
conditions; in the same-object condition, however, the target location should be coded relative to both the left/right cued location and the central fixation point. Therefore, the referential-coding account predicts that the modulation of spatial Stroop by cueing will clearly depend on the rectangles' orientation. In fact, the modulation should be the opposite for both arrangements.

\section{Method}

\section{Participants}

A total of 12 undergraduate students (4 males and 8 females) at the China Agricultural University, Beijing, China, took part in a paid experiment. All participants had normal or corrected-tonormal vision.

\section{Materials and stimuli}

Stimuli were shown on a Super VGA highresolution colour monitor. A Lenova-compatible computer running E-Prime 1.0 software controlled the presentation of stimuli, timing operations, and data collection. Participants viewed the monitor from a distance of $60 \mathrm{~cm}$ in a dimly lit room. The fixation point was a central $0.4^{\circ} \times 0.4^{\circ}$ plus sign. Each rectangle subtended $1.8^{\circ} \times 5.6^{\circ}$ with a black stroke of $0.1^{\circ}$ and was centred $1.9^{\circ}$ from the fixation point. The cue was a red square, $2.3^{\circ}$ in length and width, with a black stroke of $0.1^{\circ}$ around one end of one of the rectangles and was centred $3.6^{\circ}$ from the fixation point. The target was a black $1.0^{\circ} \times 0.4^{\circ}$ arrow, which was $2.9^{\circ}$ from the fixation point and superimposed at one end of one of the rectangles. The overall display subtended $5.6^{\circ} \times 5.6^{\circ}$.

\section{Procedure}

Each trial began with a fixation cross, which remained on the screen throughout the trial. After the cross was shown for $1,000 \mathrm{~ms}$, two rectangles appeared and also remained on the screen throughout the trial (see Figure 1 for an illustration of the trial procedure and different conditions). The two rectangles were arranged either horizontally or vertically with equal probability and were shown either to the left and right of fixation or above and below it. After they had been shown for $500 \mathrm{~ms}$, a red cue was equally likely to appear at either end of either rectangle for $100 \mathrm{~ms}$. After the cue disappeared, a black arrow target was superimposed at one of the four possible locations (the two ends of the two rectangles), with equal probability. Thus, the cue-target interval (i.e., SOA) was $100 \mathrm{~ms}$. The target remained visible until the participant responded or for $1,500 \mathrm{~ms}$ if there was no response. This terminated the current trial, and the next one began after a 500-ms intertrial interval during which the screen was white. Participants were instructed to respond to the target as quickly as possible without making errors, by pressing the " $Z$ " or the "M" key of the computer keyboard with the index fingers of either hand, depending on the direction of the arrow. The alignment of the response keys and computer screen was such that the fixation point and the midway point between the two response keys were on the participant's sagittal midline.

\section{Design}

There were two sessions of 280 trials each. Each session included six blocks: two practice blocks of 12 trials, and four test blocks of 64 trials each. In one session, the task was to press the " $Z$ " key (left response) when the arrow pointed to the left, and the "M" key (right response) when it pointed to the right, regardless of the arrow's location, while the reverse mapping was used in the other session. The order was counterbalanced across participants. Response mapping was manipulated to ensure that spatial Stroop (stimulus-stimulus interference) and not Simon (stimulus-response interference) interference was measured (Lupiáñez \& Funes, 2005). Except for this purpose, response mapping was irrelevant to the aims of our research, so data were collapsed across this manipulation.

The target arrow appeared at the same location as the cue in $25 \%$ of the trials. The arrow appeared in any of the three different types of invalid-cue trials in the remaining trials: $25 \%$ same-object trials (the target appeared at the far end of the cued rectangle), $25 \%$ equidistant different-object 
trials (the target appeared at the near end of the opposite rectangle), and 25\% far-location trials (the target appeared at the far end of the opposite rectangle-diagonally opposite the cued location). As in Macquistan (1997) and Goldsmith and Yeari (2003), the far-location trials were only included to prevent any overall correlation between the cue and target locations. Therefore, they are not included in the analyses. The target arrow pointed left on $50 \%$ of the trials and right in the remaining 50\%, thus leading to the same number of congruent (same arrow location and direction) and incongruent trials (different location and direction). Therefore, the experiment had a 2 (rectangle orientation: vertical vs. horizontal) $\times 3$ (validity: same location, same object, different object) $\times 2$ (spatial congruency: congruent vs. incongruent) design, with 32 observations per experimental condition.

\section{Results}

\section{Error rates}

Error rates for each participant were sorted into cells according to the conditions of rectangle orientation, validity, and congruency, as can be seen in Table 1.

The data were submitted to a 2 (rectangle orientation: vertical vs. horizontal) $\times 3$ (validity: same location, same object, different object) $\times 2$ (spatial congruency: congruent vs. incongruent) repeated measures analysis of variance (ANOVA). The main effect of congruency was marginally significant, $F(1,11)=4.81, p=.051$. No other effects reached significance.

\section{Reaction times}

Error trials were removed from the analysis. Medians were computed on correct reaction times (RTs) for each participant and for all experimental conditions. Means of participants' median reaction times for each condition are shown in Table 1.

The same analyses as those that were conducted on error rates were conducted on the RTs. There was a main effect of validity, $F(2,22)=52.89$, $p<.001$, with shorter RTs for same location than for same object, $t(11)=-7.02, p<.001$, and for different object, $t(11)=-8.80, p<.001$, showing a space-based cueing effect. Also, RTs were shorter for same object than for different object, $t(11)=-3.59, \quad p<.001$, showing an object-based cueing effect.

There was a marginally significant main effect of rectangle orientation, $F(1,11)=4.45, p=.059$, with shorter RTs for vertical rectangles than horizontal ones, and a main effect of spatial congruency, $F(1,11)=42.61, p<.001$, with shorter $\mathrm{RT}$ s for congruent than incongruent trials, thus showing the typical spatial Stroop effect. Furthermore, as expected, this effect was significantly modulated by validity, as revealed by the Validity $\times$ Spatial Congruency interaction, $F(2$, $22)=9.86, \quad p=.001$. Planned comparisons showed that the congruent versus incongruent effect was significant for same location, same object, and different object, $F(1,11)=15.53$, $p=.002, F(1,11)=10.94, p=.007$, and $F(1$, $11)=56.24, p<.001$, respectively.

However, congruency scores (incongruent minus congruent) for different object (40) was larger than

Table 1. Experiment 1: Mean reaction times as a function of rectangle orientation, spatial congruency, and validity conditions

\begin{tabular}{lccrrr}
\hline & \multicolumn{2}{c}{ Vertical } & & \multicolumn{2}{c}{ Horizontal } \\
\cline { 2 - 3 } Validity & Congruent & Incongruent & & Congruent & Incongruent \\
\hline Same location & $426(3.8)$ & $440(3.8)$ & & $417(2.7)$ & $438(4.0)$ \\
Same object & $459(1.5)$ & $464(2.0)$ & & $434(1.3)$ & $464(2.7)$ \\
Different object & $450(2.5)$ & $494(4.0)$ & & $442(.6)$ & $478(2.3)$ \\
\hline
\end{tabular}

Note: Mean reaction times in $\mathrm{ms}$; error percentages in parentheses. 
that for same location (18), $t(11)=3.89, p=.003$, and same object (18), $t(11)=3.54, p=.005$; the last two effects were not different from each other, $t(11)=0.032, p=.975$. The interaction between rectangle orientation, validity, and spatial congruency was not significant, $F(3,33)=2.05$, $p=.153$.

Analysing the Congruency $\times$ Validity interaction from a different perspective, the validity effect was significant in the congruent condition, $F(2,22)=18.45, p<.001$, with faster response for same location than for same object, $t(11)=-5.91, p<.001$, and for different object, $t(11)=-4.38, p<.001$; the last two conditions did not differ from each other, $t(11)=.009$, $p=.929$. The validity effect was also significant in the incongruent condition, $F(2,22)=55.40$, $p<.001$, with faster responses for same location than for same object, $t(11)=-5.39, p<.001$, and for different object, $t(11)=-11.20$, $p<.001$, and faster responses for same object than for different object, $t(11)=-4.83$, $p=.001$. Thus, both location-based and objectbased effects were observed in the incongruent condition, whereas only a location-based effect was observed in the congruent condition.

\section{Discussion}

In this experiment, responses were slower when attention had to be covertly shifted from the same location to either the same-object or the different-object location, which showed an attentional space-based effect. However, responses were also significantly delayed when attention was shifted from the same location to the different-object location, as compared to when it was shifted to the same-object location. Given that the distance and direction of between-object shifts were identical across trials to within-object shifts, the additional cost of between-object shifts must reflect a time cost for shifting attention between objects-that is, an attentional objectbased effect. Thus, both the space-based and the object-based effects obtained by Egly et al. (1994) and others were replicated in the present study. Moreover, responses were slower when the arrow's location was incongruent with its direction than when both dimensions were congruent. Therefore, a spatial Stroop effect was also obtained. Altogether, these results show that our procedure was suitable to measure space-based and object-based components of visual attention along with spatial Stroop effects and was therefore suitable to explore how they interact.

More interestingly for the aim of the study, the size of the spatial Stroop effect in the samelocation condition was almost equal to that of the same-object condition. This result clearly shows that space-based attention does not seem to modulate the spatial Stroop effect. Furthermore, the size of the spatial Stroop effect was reduced by cueing the object, with a congruency effect of $18 \mathrm{~ms}$ in the same-object and $40 \mathrm{~ms}$ in the different-object condition.

Such results constitute a perfect replication of earlier findings on exogenous cueing modulation on spatial Stroop. However, the location and object were simultaneously cued within the same condition in those studies (Danziger et al., 2001, Experiment 3; Funes et al., 2007, Experiments 1 and 2A; Lupiáñez \& Funes, 2005). An average estimation of the spatial Stroop observed in the cued location + object condition at the 100-ms SOA in those earlier studies was $15 \mathrm{~ms}$; the same average estimation for the noncued location + object was $37 \mathrm{~ms}$. This estimation is very close to the spatial Stroop effect we observed in the same-object (18 ms) and the different-object conditions (40 ms). This correspondence between location + object cueing and object-only cueing suggests that the spatial cueing modulation on the spatial Stroop effect may be fully caused by object-based attention in those studies.

Overall, these results are consistent with the predictions of the event- or object-integration account. However, they are not in agreement with the attention-shift account. According to this account, a clear sign of space-based modulation of spatial Stroop should have been observed. Finally, the pattern of results was only partially consistent with the referential-coding account. As we described in the introduction, this account predicted that spatial Stroop would decrease in 
the same-object condition, albeit only when the rectangles were vertically arranged; however, a larger congruency effect was expected to arise in the same-object condition when the rectangles were horizontally arranged. The finding of a null three-way interaction between the variables spatial congruency, validity, and rectangle orientation suggests that spatial Stroop decreased in the same-object condition compared to the different-object condition in both rectangle orientations.

Approaching the Congruency $\times$ Validity interaction from a different perspective, the location-based effect takes place in both the congruent and incongruent conditions; however, the object-based attention effect is only observed in the incongruent condition. In other words, it seems as if exogenously orienting attention towards the target location equally amplified all target dimensions-location and others (e.g., direction) - in a zoom-lens like manner; orienting attention to the object, however, seems to have a more specific effect on filtering out the irrelevant location information (i.e., a greater effect in the incongruent condition), thus decreasing the spatial Stroop effect. A similar pattern of results was observed by Shomstein and Yantis (2002), who found that responses to targets in a compatible condition between target and flanker are not slower when the target is in the different object than when it is in the same object.

\section{EXPERIMENT 2}

Funes et al. (2007) found that the peripheral cueing modulation of the spatial Stroop effect was independent of whether the cue was predictive about the target location; this conclusion was reached because a similar reduction of spatial Stroop was found following highly predictive peripheral cues and nonpredictive ones. This result was taken as evidence against an explanation focused exclusively on attentional orienting. If attentional orienting were the key source of the decrease of spatial Stroop, a greater decrease should have been observed with highly predictive cues than with nonpredictive ones. However, it remains unsolved whether the introduction of predictive cues may alter the space-based and object-based modulation of spatial Stroop differently. Experiment 2 was carried out to clarify this issue.

To evoke voluntary orienting and maintenance of attention at the cued location, targets were presented at the cued location in $70 \%$ of the trials, and participants were informed of this contingency (e.g., Abrams \& Law, 2000; Danziger \& Kingstone, 1999; Danziger et al., 2001; Funes et al., 2007). Except for the use of highly predictive cues, Experiment 2 was identical to Experiment 1.

\section{Method}

\section{Participants}

A total of 12 undergraduate students (6 males and 6 females) at China Agricultural University, Beijing, China, took part in a paid experiment. All participants had normal or corrected-tonormal vision.

\section{Procedure and materials}

The materials and procedure were identical to those of Experiment 1.

\section{Design}

There were two sessions of 660 trials each. Each session included six blocks: two practice blocks of 10 trials each and four test blocks of 160 trials each. The target arrow appeared at the cued location in $70 \%$ of the trials and at each of the other three locations in $10 \%$ of the trials. Participants were informed about this probability.

\section{Results}

\section{Error rates}

Error rates for each participant were sorted into cells according to the conditions of rectangle orientation, validity, and congruency. These mean error percentages are displayed in Table 2.

The data were subjected to a 2 (rectangle orientation: vertical vs. horizontal) $\times 3$ (validity: same location, same object, different object $) \times 2$ 
(spatial congruency: congruent vs. incongruent) repeated measures ANOVA. None of the effects reached significance.

\section{Reaction times}

Error trials were removed from the analysis. Medians were computed on correct RTs for each participant and experimental condition. Means of participants' median reaction time for each condition are shown in Table 2.

The ANOVA performed on median RTs showed a main effect of rectangle orientation, $F(1$, $11)=10.84, p=.007$, with shorter responses in the horizontal rectangle than in the vertical rectangle condition, a main effect of spatial congruency, $F(1,11)=9.98, p=.009$, and a main effect of validity, $F(2,22)=30.95, p<.001$. RTs were shorter for the same-location than for the same-object condition, $t(11)=-5.00, p<.001$, and for the different-object condition, $t(11)=$ -6.53 , showing a space-based effect, $p<.001$. RTs were shorter for same-object than for different-object conditions, $t(11)=-2.43, p=.033$, showing an object-based effect. There was an interaction between validity and spatial congruency, $F(2$, $22)=9.29, p=.001$, and between rectangle orientation and spatial Stroop, $F(1,11)=6.54$, $p=.027$. The three-way interaction between rectangle orientation, validity, and congruency also reached significance, $F(2,22)=36.03, p<.001$. To disentangle this interaction, we performed a separate ANOVA on the data from each rectangle orientation, with validity and congruency as within-participants variables.

The analysis of the data from the vertical rectangle condition revealed main effects of the two variables, validity and congruency, $F(2$, $22)=37.39, \quad p<.001, \quad F(1, \quad 11)=5.17$, $p=.044$. RTs were shorter for the same-location condition (442 ms) than for the same-object (487 ms), $t(11)=-5.55, p<.001$, and differentobject conditions (495), $t(11)=-7.41, p<.001$, showing a space-based effect. RTs were also shorter for same-object than for different-object conditions, $t(11)=-2.09, p=.060$, showing an object-based effect. Also, validity interacted with congruency, $F(2,22)=44.77, p<.001$. Planned comparisons showed that the congruent versus incongruent effect was significant in the different-object condition, $F(1,11)=36.91, p<.001$, but not in the same-location and same-object conditions, $F(1, \quad 11)=1.47, \quad p=.250, \quad F(1$, $11)=0.48, p=.505$. Congruency scores (incongruent minus congruent) for different object $(39 \mathrm{~ms})$ were higher than those for same location (6 ms), $t(11)=7.75, p<.001$, and same object $(-5 \mathrm{~ms}), t(11)=7.51, p<.001$; the latter two effects were not different from each other, $t(11)=1.60, p=.125$.

As in Experiment 1, the analysis of the Congruency $\times$ Validity interaction for the vertically arranged rectangles from a different perspective showed a significant validity effect in the congruent condition, $F(2,22)=34.51$, $p<.001$; faster responses were obtained for the samelocation condition than for the same-object, $t(11)=-6.95, \quad p<.001$, and different-object conditions, $t(11)=-4.68, p<.001$. However, responses were not faster for the same-object condition than for the different-object condition but slower, $t(11)=6.46, \quad p<.001$. The validity effect was also significant in the incongruent

Table 2. Experiment 2: Mean reaction times as a function of rectangle orientation, spatial congruency, and validity conditions

\begin{tabular}{|c|c|c|c|c|}
\hline \multirow[b]{2}{*}{ Validity } & \multicolumn{2}{|c|}{ Vertical } & \multicolumn{2}{|c|}{ Horizontal } \\
\hline & Congruent & Incongruent & Congruent & Incongruent \\
\hline Same location & $439(1.8)$ & $445(2.7)$ & $425(2.1)$ & $441(4.6)$ \\
\hline Same object & $489(3.0)$ & $484(4.3)$ & $451(3.5)$ & $495(3.8)$ \\
\hline Different object & $475(2.8)$ & $514(4.6)$ & $470(1.5)$ & $492(2.6)$ \\
\hline
\end{tabular}

Note: Mean reaction times in ms; error percentages in parentheses. 
condition, $F(2,22)=40.77, p<.001$, with faster responses for the same-location condition than for the same-object, $t(11)=-4.20, p<.001$, and different-object conditions, $t(11)=-9.74$, $p<.001$, and faster responses for same-object than for different-object conditions, $t(11)=-4.79$, $p=.001$. Thus, both location-based and objectbased effects were observed in the incongruent condition, whereas - again-only the locationbased effect was observed in the congruent condition.

The analysis of the data from the horizontal rectangle condition also revealed main effects of validity and congruency, $F(2,22)=18.84$, $p<.001, F(1,11)=11.67, p=.006$. RTs were shorter for the same-location condition than for the same-object, $t(11)=-4.22, p<.001$, and the different-object conditions, $t(11)=-5.17$, $p<.001$, showing a space-based effect. RTs were also shorter for the same-object $(473 \mathrm{~ms})$ than for the different-object conditions (481 ms), although this difference did not reach statistical significance, $t(11)=-1.41, p=.187$.

Validity interacted again with congruency, $F(3$, $33)=9.19, \quad p=.001$. Planned comparisons showed that the congruent versus incongruent effects were significant in the same-location, same-object, and different-object conditions, $F(1,11)=6.03, p=.032, \quad F(1,11)=14.11$, $p=.003, F(1,11)=8.06, p=.016$. However, contrary to what was found in the vertical-rectangle condition, congruency scores (incongruent minus congruent) for the same-object condition were higher than those for the same-location, $t(11)=3.50, p=.005$, and different-object conditions, $t(11)=2.99, p=.012$; the last two conditions were not different from each other, $t(11)=1.19, p=.260$.

Analysing the Congruency $\times$ Validity interaction for the horizontally arranged rectangles from a different perspective, the validity effect was significant in the congruent condition, $F(2$, $22)=14.26, \quad p<.001$; faster responses were obtained for the same-location condition than for the same object, $t(11)=-3.08, p=.011$, and different object conditions, $t(11)=-5.11$, $p<.001$; faster responses were obtained for same-object than for different-object conditions, $t(11)=-2.35, p=.038$. The validity effect was also significant in the incongruent condition, $F(2,22)=20.03, p<.001$, with faster responses for the same-location condition than for the same-object, $t(11)=-4.57, p<.001$, and different-object conditions, $t(11)=-4.95, p<.001$; yet, these last two effects were not different from each other, $t(11)=0.48, p=.637$. Thus, both location-based and object-based effects were observed in the congruent condition, whereas only a location-based effect was observed in the incongruent condition.

\section{Discussion}

As in Experiment 1, the space-based and objectbased attentional effects and the spatial Stroop effect were obtained.

By making the peripheral cue informative, the space-based attentional effect increased from 25 (in Experiment 1) to $40 \mathrm{~ms}$ (a similar result was observed in Danziger \& Kingstone, 1999, and Funes et al., 2007). This shows that early endogenous orienting may facilitate performance compared to the facilitation effect produced by exogenous cues, which supports the possibility that endogenous and exogenous orienting occur in parallel (see, e.g., Chica, Lupiáñez, \& Bartolomeo, 2006, Juola, Koshino, \& Warner, 1995). In contrast, the object-based effect remained the same in the two experiments (around $7 \mathrm{~ms}$ in Experiment 1 and $11 \mathrm{~ms}$ in Experiment 2), suggesting that the size of object-based attention was not affected by the predictive value of the cue. Such a result was also observed by Goldsmith and Yeari (2003), when they compared the object-based cueing effect produced by peripheral exogenous cues and centrally presented endogenous ones.

Regarding the object-based modulation of spatial Stroop, we found a considerable difference between Experiments 1 and 2. In Experiment 2, unlike Experiment 1, the interaction between validity and the spatial Stroop effect was modulated by rectangle orientation. When the rectangles were vertically arranged, the spatial Stroop effects

\section{THE QUARTERLY JOURNAL OF EXPERIMENTAL PSYCHOLOGY, 2010, 63 (3)}


were not significant in the same-location or the same-object condition, but were significant in the different-object condition. No differences in spatial Stroop were found for the same-location and same-object conditions. These results proved again that the decrease of spatial Stroop by cueing is object based but not space based. Thus, we replicated Experiment 1 when the rectangles were vertically arranged, even if the cue was informative about the target location.

Nevertheless, when the rectangles were horizontally arranged, the spatial Stroop effect was greater for the same-object condition than for the other two conditions, showing an objectbased modulation of spatial Stroop. However, contrary to Experiment 1 and the verticalrectangle condition in Experiment 2, the spatial Stroop effect increased (instead of decreasing) in the same-object condition. This pattern of results was obtained because the object-based effect was present in the congruent condition but absent in the incongruent condition. Similar results were observed in Funes et al.'s (2007) study (Experiment 2) with the use of location + object endogenous cues that were centrally presented and by Wuhr and Waszak (2003) with the typical colour-word Stroop task. They observed that colour words presented in the rectangle that colour participants were to name (i.e., at the object of attention) produced larger Stroop interference than words shown outside the object of attention.

Experiment 2 provides further evidence that peripheral cueing may modulate the spatial Stroop effect. This modulation was entirely object based, even when we introduced peripheral cues that were predictive about the target location.

\section{GENERAL DISCUSSION}

This study was conducted to explore whether the typical modulation of spatial congruency effects by peripheral cueing is object or space based. To do so, we combined the double-rectangle cueing paradigm developed by Egly et al. (1994) with the spatial Stroop task used by Lupiáñez and Funes (2005). Overall, the pattern of results that we observed in this study is clear: Once the space-based component can be measured separately from the object-based component, the previously reported attentional modulation on the spatial Stroop effect seems to be entirely object based, not space based. In Experiment 1, spatial Stroop decreased in the cued-object condition, regardless of whether the target arrow appeared at the same location or on the other side of the object (same-object condition). In Experiment 2, we replicated the effect found in Experiment 1 with the vertically arranged rectangles; spatial Stroop equally decreased in the same-location and same-object conditions. Although of a different nature, this modulation was also object based in the case of the horizontally arranged rectangles.

As described in the introduction, three possible hypotheses were proposed to explain the finding that the spatial Stroop effect is decreased by peripheral cueing: the attention-shift account (e.g., Rubichi et al., 1997), the referential-coding account (Danziger et al., 2001), and the event-integration account (e.g., Lupiáñez \& Funes, 2005).

According to the attention-shift account, once attention has been moved towards the cued location, no spatial codes are created for targets appearing at this location; therefore, no conflict should arise in the same-location condition between the irrelevant target location and its relevant direction. A problem with this account is that it predicts an equal spatial Stroop effect in the same-object condition and the different-object condition. Therefore, it fails to explain our main result - that is, the decrease of interference in same-object trials as compared to different-object trials.

A second explanation for the cueing modulation of spatial Stroop, in terms of Hommel's referential coding (1993b), is that proposed by Danziger et al. (2001). According to them, the appearance of the cue is meant to establish a reference frame for target spatial coding. If we assume that the cue "spreads" to the object in which it appears, this framework could explain the objectbased modulation we observed. Thus, when the rectangles are vertically arranged, the target 
arrow should be left/right coded relative to the central fixation cross and the lateralized cue object in the different-object condition; yet, in the same-location and same-object conditions the target arrow should be left/right coded only relative to the central cross. This would explain why the spatial congruency effects are smaller in the same-location and -object conditions than in the different-object conditions. It is worth noting that in the same-object condition the arrow target should also be up/down coded relatively to the cue. However, this dimension is orthogonal to the left/right distracting dimension. Therefore, similar spatial congruency effects are predicted in the same-location and same-object conditions in Experiments 1 and 2, as was the case.

A similar explanation is provided by the eventintegration account (Funes et al., 2007; Lupiáñez \& Funes, 2005). Given that-under appropriate spatial-temporal parameters- the target arrow is integrated within the cue representation, no extra spatial code is created whenever the target appears at the same location or object as the cue. Thus, the same spatial code generated by the cue should be used to process the target location. However, this irrelevant location dimension should have largely declined by the time the relevant direction dimension is coded. The separation in time of these two perceptual codes may then underlie the decrease in the spatial congruency effect in same-location or same-object trials. In contrast, when there is no correspondence-as happens in the different-object condition-cue-target event integration should not occur; therefore, spatial Stroop should not decrease, as a spatial code should be generated when the target appears, thus interfering with target direction coding.

When the rectangles were horizontally arranged, however, a considerably different pattern of results was observed depending on whether the cue was predictive or not. Whereas in Experiment 1, with nonpredictive cues, spatial Stroop similarly decreased in the same-object condition as with vertically arranged rectangles, the opposite pattern was observed in Experiment 2, with predictive cues. In this case, spatial Stroop increased-rather than decreasing-in the sameobject condition (i.e., the location opposite the cue within the same object) as compared to the same-location condition. Again, if we assume that the cue "spreads" within the cued object, the referential-coding as well as the event-integration accounts could easily explain the pattern of results observed with nonpredictive cues. However, both of these accounts might have difficulty explaining the pattern of results with predictive cues, unless it is considered that cue predictability might change the way each location within the cued object is coded. On the surface, the decrease of spatial Stroop by cueing seems to be exclusively due to exogenous and object-based attention (Funes et al., 2007; this paper), thus supporting the independence of exogenous and endogenous attention (Funes et al., 2007; see Klein \& Shore, 2000, for a review). Yet, it seems plausible that the latter may modulate the way exogenous attention affects processing. In fact, a qualitatively different pattern of results can be observed with predictive versus nonpredictive peripheral cues (Prinzmetal, McCool, \& Park, 2005).

Thus, with nonpredictive cues, we might assume that each side of the rectangle is similarly coded as belonging to the cued object. However, with predictive cues, the cued end of the rectangle might be coded more specifically as different from the uncued end (after all, it is at the cued end that the target is most likely to appear). Thus, when the target appears at the opposite (uncued) end of the rectangle, it will be left/right coded relative to the cue. Appearing to the left or right of the cue, but inside the cued object, might lead to a greater generation of a left/right code than appearing outside the cued object, thus explaining why spatial Stroop was greater in the same-object condition of Experiment 2 with horizontally arranged rectangles. Thus, although this later explanation is somehow ad hoc, both the referential-coding and the event-integration accounts may explain the overall pattern of results observed in our two experiments; indeed, they both assume that exogenous cueing effects in general (and those on spatial Stroop in particular) are mediated by object-based representations. 
Lupiáñez and colleagues (Funes et al., 2007; Lupiáñez et al., 2005) rather preferred the eventintegration account, as it was able to explain other results that proved difficult to explain by the referential-coding account. For example, the latter account seems to have difficulty explaining why the reduction of spatial Stroop at the cued location/object seems to be greater at short SOAs (Funes et al., 2007; Lupiáñez \& Funes, 2005) or disappears when a distractor is shown at the opposite location to the target (Funes et al., 2008). Further research is necessary to specifically distinguish both accounts.

Altogether, we may conclude that the modulation of attention over the generation of spatial codes that interfere with the processing of the target direction in the spatial Stroop task seems to be related to object-based attention. These object-based effects seem to be involuntary, characteristic of exogenous attention (Goldsmith \& Yeari, 2003) and triggered by peripheral cues, although endogenous attention seems to be able to modulate them.

Original manuscript received 11 September 2007 Accepted revision received 12 February 2009 First published online 29 July 2009

\section{REFERENCES}

Abrams, R. A., \& Law, M. B. (2000). Object-based visual attention with endogenous orienting. Perception E Psychophysics, 62, 818-833.

Chica, A. B., Lupiáñez, J., \& Bartolomeo, P. (2006). Dissociating inhibition of return from endogenous orienting of spatial attention: Evidence from detection and discrimination tasks. Cognitive Neuropsychology, 23(7), 1015-1034.

Danziger, S., \& Kingstone, A. (1999). Unmasking the inhibition of return phenomenon. Perception $\mathcal{E}^{\circ}$ Psychophysics, 61, 1024-1037.

Danziger, S., Kingstone, A., \& Ward, R. (2001). Environmentally defined frames of reference: Their sensitivity to spatial cues and attention, and their time course. Journal of Experimental Psychology: Human Perception and Performance, 27, 494-503.

Egly, R., Driver, J., \& Rafal, R. D. (1994). Shifting visual attention between objects and locations:
Evidence from normal and parietal lesion subjects. Journal of Experimental Psychology: General, 123, 161-177.

Funes, M. J., \& Lupiáñez, J. (2003). La teoría atencional de Posner: Una tarea para medir las funciones atencionales de orientación, alerta y control cognitivo y la interacción entre ellas [Posner's theory of attention: A task to measure the attentional functions of orienting, alerting and cognitive control and the interactions between them]. Psicothema, 15, 260-266.

Funes, M. J., Lupiáñez, J., \& Milliken, B. (2005). The role of spatial attention and other processes on the magnitude and time course of cueing effects. Cognitive Processing-International Quarterly of Cognitive Science, 6, 98-116.

Funes, M. J., Lupiáñez, J, \& Milliken, B. (2007). Separate mechanisms recruited by exogenous and endogenous spatial cues: Evidence from a spatial Stroop paradigm. Journal of Experimental Psychology: Human Perception and Performance, 33(2), 348-262.

Funes, M. J., Lupiáñez, J, \& Milliken, B. (2008). The modulation of exogenous spatial cueing on spatial Stroop interference: Evidence of a set for "cuetarget event segregation”. Psicológica, 29, 65-95.

Goldsmith, M., \& Yeari, M. (2003). Modulation of object-based attention by spatial focus under endogenous and exogenous orienting. Journal of Experimental Psychology: Human Perception and Performance, 29(5), 897-918.

Hommel, B. (1993a). The relationship between stimulus processing and response selection in the Simon task: Evidence for a temporal overlap. Psychological Research, 55, 280-290.

Hommel, B. (1993b). The role of attention for the Simon effect. Psychological Research, 55, 208-222.

Ivanoff, J., Klein, R. M., \& Lupiáñez, J. (2002). Inhibition of return interacts with the Simon effect: An omnibus analysis and its implications. Perception E Psychophysics, 64, 318-327.

Jonides, J., \& Yantis, S. (1988). Uniqueness of abrupt visual onset in capturing attention. Perception $\mathcal{F}^{\circ}$ Psychophysics, 43, 346-354.

Juola, J. F., Koshino, H., \& Warner, C. B. (1995). Tradeoffs between attentional effects of spatial cues and abrupt onsets. Perception and Psychophysics, 57, 333-342.

Kahneman, D., Treisman, A., \& Gibbs, B. (1992). The reviewing of object files: Object-specific integration of information. Cognitive Psychology, 24, 175-219.

Klein, R. M., \& Shore, D. I. (2000). Relations among modes of visual orienting. In S. Monsell \& J. Driver (Eds.), 
Attention \& Performance XVIII: Control of cognitive processes (pp. 195-208). Cambridge, MA: The MIT Press.

Lu, C.-H., \& Proctor, R. W. (1994). Processing of an irrelevant location as a function of the relevant stimulus dimension. Journal of Experimental Psychology: Human Perception and Performance, 20, 286-298.

Lu, C.-H., \& Proctor, R. W. (1995). The influence of irrelevant location information on performance: A review of the Simon effect and congruency effects. Psychonomic Bulletin E Review, 2, 174-207.

Lupiáñez, J., \& Funes, M. J. (2005). Peripheral spatial cues modulate spatial Stroop interference: Analyzing the "locus" of the cueing modulation. European Journal of Experimental Psychology, 17, 727-752.

Lupiáñez, J., \& Milliken, B. (1999). Inhibition of return and the attentional set for integrating versus differentiating information. The Journal of General Psychology, 126(4), 392-418.

Lupiáñez, J., Milliken, B., Solano, C., Weaver, B., \& Tipper, S. P. (2001). On the strategic modulation of the time course of facilitation and inhibition of return. The Quarterly Journal of Experimental Psychology, 54(3), 753-773.

Macquistan, A. D. (1997). Object-based allocation of visual attention in response to exogenous, but not endogenous, spatial precues. Psychonomic Bulletin $\xi^{\circ}$ Review, 4, 512-515.

O'Leary, M., \& Barber, P. (1993). Interference effects in the Stroop and Simon paradigms. Journal of Experimental Psychology: Human Perception and Performance, 19, 830-844.

Prinzmetal, W., McCool, C., \& Park, S. (2005). Attention: Reaction time and accuracy reveal different mechanisms. Journal of Experimental Psychology: General, 134(1), 73-92.

Proctor, R. W., Lu, C. H., \& Van Zandt, T. (1992). Enhancement of the Simon effect by response precuing. Acta Psychologica, 81, 53-74.

Rubichi, S., Nicoletti, R., Iani, C., \& Umilta, C. (1997). The Simon effect occurs in relation to the direction of an attention shift. Journal of
Experimental Psychology: Human Perception and Performance, 5, 1353-1364.

Rubichi, S., Vu, K.-P. L., Nicoletti, R., \& Proctor, R. W. (2006). Spatial coding in two dimensions. Psychonomic Bulletin \& Review, 13, 201-216.

Shomstein, S., \& Yantis, S. (2002). Object-based attention: Sensory modulation or priority setting? Perception E Psychophysics, 64, 41-51.

Stoffer, T. H. (1991). Attentional focusing and spatial stimulus-response compatibility. Psychological Research, 53, 127-135.

Taylor, T. L., \& Ivanoff, J. (2005). Inhibition of return and repetition priming effects in localization and discrimination tasks. Canadian Journal of Experimental Psychology, 59, 75-89.

Verfaellie, M., Bowers, D., \& Heilman, K. M. (1988). Attentional factors in the occurrence of stimulusresponse compatibility effects. Neuropsychologia, 26, 435-444.

Walley, R. E., McLeod, B. E., \& Weiden, T. D. (1994). Increased attention to the irrelevant dimension increases interference in a spatial Stroop task. Canadian Journal of Experimental Psychology, 48, 467-492.

Weaver, B., Lupiáñez, J., \& Watson, F. L. (1998). The effects of practice on object-based, location-based, and static-display inhibition of return. Perception $\xi^{\circ}$ Psychophysics, 60, 993-1003.

Weeks, D. J., Chua, R., \& Hamblin, K. (1996). Attention shifts and the Simon effect: A failure to replicate Stoffer (1991). Psychological Research, 58, 246-253.

Wuhr, P., \& Waszak, F. (2003). Object-based attentional selection can modulate the Stroop effect. Memory E Cognition, 31(6), 983-994.

Yantis, S., \& Jonides, J. (1996). Attentional capture by abrupt onsets: New perceptual objects or visual masking? Journal of Experimental Psychology: Human Perception and Performance, 22, 1505-1513.

Zimba, L. D., \& Brito, C. F. (1995). Attention precuing and Simon effects: A test of the attention-coding account of the Simon effect. Psychological Research, 58, 102-118. 